\title{
COSTO OPORTUNIDAD EN LA EMBARAZADA QUE DEMANDA ATENCIÓN EN PRIMER NIVEL DE ATENCIÓN
}

\author{
Laura Cu Flores ${ }^{1,2}$, Enrique Villarreal Ríos ${ }^{1}$, Emma Vargas Daza ${ }^{1, a}$, Liliana Galicia \\ Rodríguez ${ }^{1, b}$, Lidia Martínez González ${ }^{1, a}$. \\ ${ }^{1}$ Unidad de Investigación Epidemiológica y en Servicios de Salud Querétaro, ${ }^{2}$ Unidad de Medicina Familiar №16 \\ "Dr. Arturo Guerrero Ortíz", Instituto Mexicano del Seguro Social, Querétaro, México.
}

a Licenciada en Enfermería. ${ }^{b}$ Licenciada en Informática

\section{RESUMEN}

Objetivo: Determinar el costo oportunidad de la embarazada que demanda atención en primer nivel de atención. Método: Estudio de costo oportunidad en la embarazada atendida en consulta de Medicina Familiar, Laboratorio y Farmacia, que acudieron solas o acompañadas a 3 unidades de Medicina Familiar. Se estudiaron 807 embarazadas. La técnica muestral contempló conglomerados (Unidad Médica), estratos (consultorio) y al interior por cuota. Se estudió edad, estado civil, ocupación y número de acompañantes. El costo oportunidad se evaluó a través de tiempo invertido y el costo por minuto; se estimó para el traslado, espera y atención en los tres servicios señalados, para la paciente y acompañante. Se utilizó porcentajes, promedios e intervalos de confianza del $95 \%$. Resultados: El costo oportunidad de la embarazada sola en consulta de Medicina Familiar es \$46,48 (IC95\% 41,68-51,27), del acompañante 1 \$49,91 (IC 95\% 41,4958,39 ) y acompañante $2 \$ 9,84$ (IC95\% 4,05-15,99); cuando la embarazada acude con un acompañante el costo oportunidad es de $\$ 95,62$ y con dos acompañantes $\$ 106,23$. El costo oportunidad de la embarazada sola en Farmacia es $\$ 22,14$ y en Laboratorio $\$ 34,82$. El costo oportunidad ajustado por número de acompañantes en consulta de Medicina Familiar es $\$ 77,14$; si utiliza en la misma visita la consulta de Medicina Familiar, Laboratorio y Farmacia es de $\$ 104,20$. Conclusión: El costo oportunidad en la paciente embarazada que demanda atención en primer nivel tiene variaciones importantes determinadas por el número de servicios que utilice y la presencia de acompañantes.

\section{PALABRAS CLAVE: Economía de la salud, costo oportunidad, control prenatal, medicina familiar}

\section{SUMMARY}

Objective: To determine the cost opportunity for pregnant women who receive first-level health care. Methods. Cost opportunity study including 807 pregnant females who received first-level care in the family medicine, laboratory and pharmacy areas. The sample was distributed by medical unit and stratified by office. Variables included age, marital status, occupation, number of accompanying; cost opportunity was estimated for transfers, waiting and care time in the mentioned service areas. Cost opportunity was calculated for the patient and for those persons who accompanied the patient and included the time invested and cost per minute. Statistical analysis included averages and $95 \%$ confidence intervals. Results. Cost opportunity for pregnant alone in family medicine is $\$ 46.48$ (IC95\% 41.68-51.27), companion $1 \$ 49.91$ (IC95\% 41.4958.39) and companion 2 \$9.84 (IC95\% 4.05-15.99); for pregnant women and one companion, cost opportu- 
nity is $\$ 95.62$ and whit two companions $\$ 106.23$. Cost opportunity for pregnant women alone in pharmacy is $\$ 22.14$ and laboratory $\$ 34.82$. Cost opportunity adjusted by companions in family medicine is $\$ 77.14$, if used in the same visit family medicine, laboratory and pharmacy is $\$ 104.20$. Conclusion. Cost opportunity for a pregnant woman who receives first-level care it has significant variations determined by the number of services that use and the presence of companions.

\section{KEY WORDS. Health economics, cost opportunity, prenatal care, family medicine}

\section{INTRODUCCIÓN}

La economía aplicada al área de la salud a través de la evaluación económica tiene como objetivo apoyar la toma de decisiones y elegir diferentes alternativas en el empleo de los recursos (1-3). Dentro de los estudios de costos se plantean dos líneas; los costos de producción de servicios para el proveedor y los costos de búsqueda y obtención de servicios para el consumidor (4), éste último se ubica en el análisis costo oportunidad que aborda los beneficios que se dejan de obtener mediante la mejor alternativa al alcance, son las pérdidas que supone la asignación de recursos a una actividad elegida $(3,5)$.

El indicador más utilizado en esta evaluación es el tiempo, al establecer que la inversión de tiempo supone un costo para el afectado y dicho costo del tiempo una pérdida que afecta a los ingresos o a la producción de una persona (tiempo de trabajo remunerado). En el caso de la persona con trabajo no remunerado existen modelos para darle un valor a ese tiempo; uno se basa en el costo de reemplazar la actividad que se deja de realizar, el otro es el costo de oportunidad del tiempo libre, medido por los ingresos que se dejan de percibir al no trabajar más que un cierto número de horas (5-6).

Los análisis económicos por grupos específicos de población, hombres, niños, mujeres, adultos mayores plantean diferentes panoramas en relación a los costos que se pueden generar (7). En el caso de las mujeres se estima que en México se presentan 2,1 millones de embarazos al año (8). En el año 2008 la institución con mayor número de derechohabientes proporcionó 3.742.141 consultas a embarazadas; con un promedio de 6 consultas por embarazada durante la gestación (9). En el primer nivel de atención el servicio con mayor demanda corresponde a la Consulta de Medicina Familiar, $85,5 \%$ de las embarazadas hacen uso de él, estableciéndose la supervisión del embarazo normal como su principal motivo de atención (10).

Dada la alta demanda de atención médica en este grupo, se considera importante determinar el costo oportunidad de la embarazada que solicita atención en el Primer Nivel de Atención.

\section{MATERIAL Y MÉTODOS}

Estudio de costo oportunidad realizado en la embarazada que demandó atención en los servicios de Consulta de Medicina Familiar, Laboratorio y Farmacia del primer nivel de atención, de las Unidades de Medicina Familiar № 9, 13 y 16 del Instituto Mexicano del Seguro Social en la Ciudad de Querétaro, México, realizado de mayo de 2008 a junio de 2009. Se incluyeron embarazadas que acudieron solas o acompañadas, aceptantes de la entrevista, excluyendo a la que solicitó atención de urgencia.

El tamaño de muestra se calculó mediante la fórmula de promedios para población infinita con nivel de confianza del 95\% y desviación estándar para Consulta de Medicina Familiar de 2,24, para Laboratorio de 2,50 y para Farmacia de 0,12 . Se obtuvo un tamaño de muestra de 269 embarazadas para cada uno de los servicios propuestos (Consulta de Medicina Familiar, Laboratorio y Farmacia) para un total de 807 embarazadas. Las 269 pacientes embarazadas de cada servicio (Consulta de Medicina Familiar, Laboratorio y Farmacia) se dividieron de manera proporcional al total de población usuaria de cada una de las unidades médicas, correspondiendo a la Unidad de Medicina Familiar №9, 78 pacientes para cada uno de los servicios; a la Unidad de Medicina Familiar №13, 102 pacientes para cada uno de los servicios; y a la Unidad de Medicina Familiar №16, 89 pacientes para cada uno de los servicios.

En un primer momento la técnica muestral fue por conglomerados (Unidad de Medicina Familiar), al interior de cada unidad se estratificaron proporcionalmente por consultorio, al interior de éstos la elección fue por cuota, considerando como marco muestral el listado nominal de pacientes registrados para ser atendidos en el consultorio el día de la entrevista. La paciente identificada en la consulta de Medicina Familiar fue la misma que la entrevistada 
en Farmacia. Para Laboratorio la técnica muestral fue por cuota, empleando como marco muestral el listado nominal de pacientes que el día de la entrevista se registraron para ser atendidos en el servicio de Laboratorio.

En la paciente las variables sociodemográficas estudiadas fueron edad, estado civil, ocupación y número de acompañantes; para el (los) acompañante(s) se analizó la ocupación. El costo oportunidad se operacionalizó a través de tiempo invertido y el costo por minuto; se estimó para el traslado, espera y atención en los tres servicios señalados, para la paciente y acompañante(s).

Tiempo de traslado, espera y atención:

- Tiempo de traslado: Promedio medido en minutos desde la sede de partida a la unidad médica multiplicado por dos para considerar el regreso.

- Tiempo de espera: Promedio medido en minutos desde el momento de llegada a la unidad médica hasta que recibió atención en Medicina Familiar y Laboratorio, para Farmacia desde el momento de llegada a dicho servicio hasta su atención.

- Tiempo de atención: Promedio medido en minutos desde el momento que inició hasta que finalizó la misma en cada uno de los servicios.

Costo del minuto:

- Actividad laboral remunerada: Se estimó preguntando directamente el salario del paciente ya sea por día, semana, quincena ó mes; esta cantidad se dividió entre el total de días (siete), (quince), (treinta), y posteriormente entre las horas laboradas al día (ocho), y posteriormente estimada por minuto (sesenta).

- Actividad laboral no remunerada: Se estimó a partir del costo promedio del minuto de la población con actividad remunerada.

- Estudiante: El costo del minuto para el estudiante se estimó en pesos mexicanos considerando la inversión familiar promedio anual $(\$ 733,17)$ para el sostenimiento por alumno reportado por la Secretaría de Educación Pública. Incluyó el gasto en uniformes $(\$ 319,39)$, cuotas $(\$ 162,58)$ y paquete de útiles y libros $(\$ 251,20)$; dividido entre el número de días escolares (260), entre las horas que acuden a la escuela al día (cinco) y posteriormente estimado por minuto (11).

- Guardería: El costo del minuto para el acompañante que acude a guardería se estimó en pesos mexicanos a partir de los indicadores de desempeño y costo de los servicios de guardería para el 2001(12) y actualizado al $2008(\$ 5.299,57)$ de acuerdo al reporte anual de inflación del Banco de México (13). Dividido entre los días hábiles al año (trescientos sesenta), dividido entre las horas que labora al día una madre trabajadora (ocho) y poste- riormente estimado por minuto.

Costo oportunidad:

- Costo oportunidad promedio del traslado del paciente y sus acompañantes: Calculado en base al tiempo estimado en el traslado multiplicado por el respectivo costo en minuto.

- Costo oportunidad promedio de la espera del paciente y sus acompañantes. Se estimó en base al tiempo de espera para recibir atención, multiplicado por el respectivo costo en minuto.

- Costo oportunidad promedio de la atención del paciente y sus acompañantes: Se estimó en base al tiempo de atención multiplicado por el respectivo costo en minuto.

- Costo oportunidad promedio por servicio: Se estimó para la Consulta de Medicina Familiar, Laboratorio y Farmacia, para el paciente y sus acompañantes a partir de la suma del costo oportunidad promedio en traslado $\left(\mathrm{C} \mu_{\mathrm{t}}\right)$, espera $\left(\mathrm{C} \mu_{\mathrm{e}}(\mathrm{mf}) ; \mathrm{C} \mu_{\mathrm{e}}(\mathrm{f})\right.$;

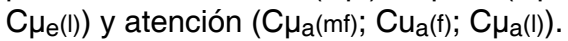

Costo oportunidad gama de escenarios:

- Costo oportunidad promedio escenario Medicina Familiar: Se estimó el costo oportunidad promedio de Medicina Familiar sumando el costo promedio de traslado, más el costo promedio de espera y atención en el servicio.

\begin{tabular}{|c|c|}
\hline $\mathrm{CO} \mu(\mathrm{mf})$ paciente solo= & $\begin{array}{l}{\left[\mathrm{CO}_{\mu \mathrm{t}}+\mathrm{C}_{\mu \mathrm{e}}(\mathrm{mf})+\mathrm{C}_{\mu \mathrm{a}}(\mathrm{mf})\right]} \\
\text { paciente }\end{array}$ \\
\hline $\begin{array}{l}\mathrm{CO} \mu(\mathrm{mf}) \text { paciente }+ \\
\text { acompañante } 1=\end{array}$ & $\begin{array}{l}{\left[\mathrm{CO}_{\mu \mathrm{t}+} \mathrm{C} \mu \mathrm{e}(\mathrm{mf})+\mathrm{C} \mu \mathrm{a}(\mathrm{mf})\right] \text { paciente }+} \\
{\left[\mathrm{CO}_{\mu \mathrm{t}+} \mathrm{C}_{\mu \mathrm{e}}(\mathrm{mf})+\mathrm{C} \mu \mathrm{a}(\mathrm{mf})\right]} \\
\text { acompañante } 1\end{array}$ \\
\hline $\begin{array}{l}\mathrm{CO} \mu(\mathrm{mf}) \text { paciente }+ \\
\text { acompañante } 1 \text { y } 2=\end{array}$ & $\begin{array}{l}{[\mathrm{C} \mu \mathrm{t}+\mathrm{C} \mu \mathrm{e}(\mathrm{mf})+\mathrm{C} \mu \mathrm{a}(\mathrm{mf})] \text { paciente }+} \\
{\left[\mathrm{C} \mu \mathrm{t}+\mathrm{C} \mu \mathrm{e}(\mathrm{mf})+\mathrm{C}_{\mu \mathrm{a}(\mathrm{mf})] \text { acompañante }}\right.} \\
1+[\mathrm{C} \mu \mathrm{t}+\mathrm{C} \mu \mathrm{e}(\mathrm{mf})+\mathrm{C} \mu \mathrm{a}(\mathrm{mf})] \\
\text { acompañante } 2\end{array}$ \\
\hline
\end{tabular}

- Costo oportunidad promedio escenario Farmacia: Se estimó el costo oportunidad promedio de Farmacia sumando el costo promedio de traslado; más el costo promedio de espera y atención en el servicio.

\begin{tabular}{|c|c|}
\hline $\mathrm{CO} \mu(f)$ paciente solo $=$ & {$[\mathrm{C} \mu \mathrm{t}+\mathrm{C} \mu \mathrm{e}(\mathrm{f})+\mathrm{C} \mu \mathrm{a}(\mathrm{f})]$ paciente } \\
\hline $\begin{array}{l}\mathrm{CO} \mu(f) \text { paciente + } \\
\text { acompañante } 1=\end{array}$ & $\begin{array}{l}{\left[C_{\mu t}+C_{\mu e}(f)+C \mu a(f)\right] \text { paciente }+} \\
{\left[C_{\mu t}+C_{\mu e}(f)+C_{\mu a(f)]}\right] \text { acompañante } 1}\end{array}$ \\
\hline $\begin{array}{l}\text { CO } \mu(f) \text { paciente }+ \\
\text { acompañante } 1 \text { y } 2=\end{array}$ & 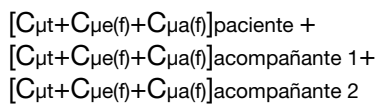 \\
\hline
\end{tabular}


- Costo oportunidad promedio escenario Laboratorio: Se estimó el costo oportunidad promedio de Laboratorio sumando el costo promedio de traslado; más el costo promedio de espera y atención en servicio.

\begin{tabular}{|c|c|}
\hline $\mathrm{CO}_{\mu}(\mathrm{l})$ paciente solo= & {$\left[\mathrm{C}_{\mu t}+\mathrm{C}_{\left.\mu \mathrm{e}(\mathrm{l})+\mathrm{C}_{\mu \mathrm{a}}(\mathrm{l})\right] \text { paciente }}\right.$} \\
\hline $\begin{array}{l}\mathrm{CO} \mu(()) \text { paciente + } \\
\text { acompañante } 1=\end{array}$ & 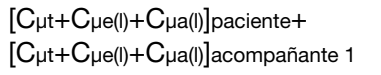 \\
\hline $\begin{array}{l}\mathrm{CO} \mu(() \text { paciente }+ \\
\text { acompañante } 1 \text { y } 2=\end{array}$ & 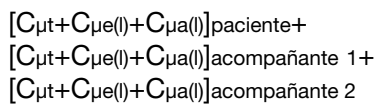 \\
\hline
\end{tabular}

- Costo oportunidad promedio escenario Medicina Familiar y Farmacia: Se estimó el costo promedio de Medicina Familiar y Farmacia sumando el costo promedio de traslado; más el costo promedio de espera y atención en Medicina Familiar y Farmacia.

\begin{tabular}{|c|c|}
\hline $\mathrm{CO} \mu(\mathrm{mf}, \mathrm{f})$ paciente solo $=$ & 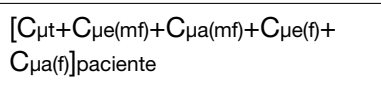 \\
\hline $\begin{array}{l}\mathrm{CO} \mu(\mathrm{mf}, \mathrm{f}) \text { paciente }+ \\
\text { acompañante } 1=\end{array}$ & 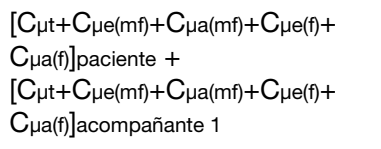 \\
\hline $\begin{array}{l}\mathrm{CO} \mu(\mathrm{mf}, \mathrm{f}) \text { paciente }+ \\
\text { acompañante } 1 \text { y } 2=\end{array}$ & $\begin{array}{l}{\left[C_{\mu t}+C_{\mu e}(m f)+C_{\mu a}(m f)+C_{\mu e}(f)+C(f)\right]} \\
\text { paciente+ } \\
{\left[C_{\mu t}+C_{\mu e}(m f)+C_{\mu a}(m f)+C \mu e(f)+a(f)\right]} \\
\text { acompañante } 1+ \\
{\left[C_{\mu t+C}+C_{\mu e}(m f)+C \mu a(m f)+C \mu e(f)+a(f)\right]} \\
\text { acompañante 2 }\end{array}$ \\
\hline
\end{tabular}

- Costo oportunidad promedio escenario Medicina Familiar y Laboratorio: Se estimó el costo oportunidad promedio de Medicina Familiar y Laboratorio sumando el costo promedio de traslado; más el costo promedio de espera y atención en Medicina Familiar y Laboratorio.

\begin{tabular}{|c|c|}
\hline $\mathrm{CO} \mu(\mathrm{mf}, \mathrm{l})$ paciente solo= & $\begin{array}{l}{[\mathrm{C} \mu \mathrm{t}+\mathrm{C} \mu \mathrm{e}(\mathrm{mf})+\mathrm{C} \mu \mathrm{a}(\mathrm{mf})+\mathrm{C} \mu \mathrm{e}(\mathrm{l})+} \\
\mathrm{C} \mu \mathrm{a}(\mathrm{l})] \text { paciente }\end{array}$ \\
\hline $\begin{array}{l}\mathrm{CO} \mu(\mathrm{mf}, \mathrm{l}) \text { paciente }+ \\
\text { acompañante } 1=\end{array}$ & 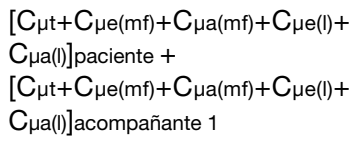 \\
\hline $\begin{array}{l}\mathrm{CO} \mu(\mathrm{mf}, \mathrm{l}) \text { paciente }+ \\
\text { acompañante } 1 \text { y } 2=\end{array}$ & 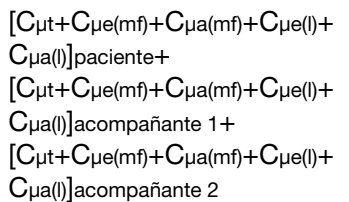 \\
\hline
\end{tabular}

- Costo oportunidad promedio escenario Farmacia y Laboratorio: Se estimó en base al costo promedio de traslado; más el costo promedio de espera y atención en Farmacia; más el costo promedio de espera y atención en Laboratorio.

\begin{tabular}{|c|c|}
\hline $\mathrm{CO} \mu(\mathrm{f}, \mathrm{l})$ paciente solo $=$ & $\begin{array}{l}{\left[C_{\mu t}+C_{\mu e}(f)+C \mu a(f)+C \mu e(l)\right.} \\
\left.+C_{\mu a}(l)\right] \text { paciente }\end{array}$ \\
\hline $\begin{array}{l}\text { COH(f,l)paciente + } \\
\text { acompañante } 1=\end{array}$ & 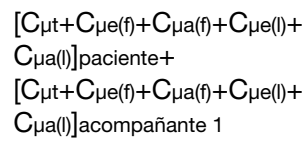 \\
\hline $\begin{array}{l}\mathrm{CO} \mu(\mathrm{f}, \mathrm{l}) \text { paciente }+ \\
\text { acompañante } 1 \text { y } 2=\end{array}$ & 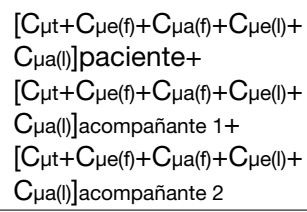 \\
\hline
\end{tabular}

- Costo oportunidad promedio escenario Medicina Familiar, Farmacia y Laboratorio: Se estimó en base al costo promedio de traslado; más el costo promedio de espera y atención en Medicina Familiar; más el costo promedio de espera y atención en Farmacia; más el costo promedio de espera y atención en Laboratorio.

\begin{tabular}{|c|c|}
\hline $\mathrm{CO} \mu(\mathrm{mf}, \mathrm{f}, \mathrm{l})$ paciente solo= & $\begin{array}{l}{\left[C_{\mu t}+\mathrm{C} \mu \mathrm{e}(\mathrm{mf})+\mathrm{C} \mu \mathrm{a}(\mathrm{mf})+\mathrm{C} \mu \mathrm{e}(\mathrm{f})+\right.} \\
\mathrm{C} \mu \mathrm{a}(\mathrm{f})+\mathrm{C}_{\mu \mathrm{e}(\mathrm{l})+\mathrm{C} \mu \mathrm{a}(\mathrm{l})] \text { paciente }}\end{array}$ \\
\hline $\begin{array}{l}\text { CO } \mu(\text { mf,f,l)paciente }+ \\
\text { acompañante } 1=\end{array}$ & $\begin{array}{l}{\left[C_{\mu t}+C_{\mu e}(m f)+C_{\mu a}(m f)+C_{\mu e}(f)+\right.} \\
\left.C \mu a(f)+C_{\mu e}(l)+C_{\mu a}(l)\right] \text { paciente+ } \\
{\left[C_{\mu t}+C_{\mu e}(m f)+C \mu a(m f)+C \mu e(f)+\right.} \\
\left.C \mu a(f)+C \mu e(l)+C_{m a}(l)\right] \text { acompañante } 1\end{array}$ \\
\hline $\begin{array}{l}\text { CO } \mu(m f, f, l) \text { paciente }+ \\
\text { acompañante } 1 \text { y } 2=\end{array}$ & 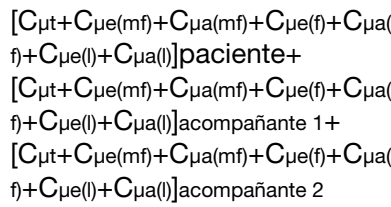 \\
\hline
\end{tabular}

- Costo oportunidad promedio ajustado: Se estimó con el costo oportunidad promedio por servicio ajustado por el porcentaje de población de pacientes que acuden con o sin acompañantes.

El análisis estadístico incluyó promedios, porcentajes e intervalos de confianza del 95\%. Para comparaciones se estima la equivalencia de $1 \$ \cup S=12,9$ \$MXN.

\section{RESULTADOS}

El promedio de edad de las pacientes embarazadas fue de 26,75 años (IC 95\%, 26,36-27,14), 
predomina el estado civil casada en $61,4 \%$ (IC $95 \%, 57,10-65,70)$, ocupación ama de casa $31,9 \%$ (IC 95\%, 26,20-37,60) y empleada $31,0 \%$ (IC 95\%, 25,30-36,70). El 40,1\% (IC 95\%, 38,4-45,4) acuden solas; $52,1 \%$ (IC 95\%, 47,30-56,90) con un acompañante, y con dos acompañantes $7,8 \%$ (IC 95\%, $1,20-14,40)$.

En el acompañante 1 predomina la ocupación empleado 28\% (IC 95\%, 20,40-35,60); y en el acompañante 2, estudiantes 52,4\% (IC 95\%, 35,4069,40).

En Consulta de Medicina Familiar el costo oportunidad para la paciente es de $\$ 46,48$ (IC 95\%, $41,68-51,27$ ), en el acompañante $1 \$ 49,91$ (IC $95 \%, 41,49-53,39$ ) y en el acompañante $2 \$ 9,84$ (IC $95 \% 4,05-15,99)$. El costo oportunidad en traslado espera y atención para la paciente y acompañantes en la consulta de Medicina Familiar se presenta en la Tabla I.

El costo oportunidad en Farmacia para la paciente es \$22,14 (IC 95\%, 20,46-23,81), para el acompañante 1 \$23,93 (IC 95\%, 21,43-26,44) y para el acompañante $2 \$ 7,73$ (IC 95\% 4,13-11,33). El costo oportunidad en traslado, espera y atención en Farmacia para la paciente y acompañantes se muestra en la Tabla II.

En Laboratorio para la paciente el costo oportunidad es de $\$ 34,82$ (IC $95 \%, 32,26-37,42$ ), para el acompañante $1 \$ 38,13$ (IC 95\% 33,95-42,33) y acompañante $2 \$ 12,57$ (IC 95\% 5,1-19,37). El costo oportunidad de traslado espera, atención y totales muestra en la Tabla III.

El costo oportunidad promedio para el escenario de Farmacia cuando la paciente acude sola es de $\$ 22,14$, y para el escenario Medicina Familiar, Farmacia y Laboratorio cuando la paciente acude con dos acompañantes el costo oportunidad es de $\$ 145,35$. En la Tabla IV se presenta el costo oportunidad para las combinaciones de servicios cuando la paciente acude sola, con un acompañante o con dos acompañantes.

El costo oportunidad promedio ajustado por el porcentaje de pacientes que acuden solas o acompañadas, es de $\$ 77,14$ en la Consulta de Medicina Familiar, de $\$ 58,64$ en Laboratorio y de $\$ 104,20$ en Consulta de Medicina familiar, Farmacia y Laboratorio. En la Tabla V se presenta el costo oportunidad para cada uno de las combinaciones de servicios.

\section{DISCUSIÓN}

La importancia del estudio del costo oportunidad de la mujer embarazada radica en el alto número de embarazos que se registran anualmente, aunado a ello la demanda inducida aunque necesaria en este grupo, puesto que es incuestionable la importancia del control prenatal.

Estudiar el costo oportunidad aporta información que en un primer momento identifica el panorama desde la perspectiva económica, información que en un segundo momento puede ser utilizada por el tomador de decisiones en la planeación de los servicios de salud considerando la perspectiva de la población, perspectiva no muy frecuentemente abordada.

Tradicionalmente el costo de la atención se establece desde el enfoque de la institución o del sistema de salud, aunado a ello es una realidad la tendencia de los sistemas de salud a transferir o compartir responsabilidad en la atención del paciente con la familia o el grupo social cercano. Ante este panorama resalta la importancia del abordaje propuesto, en este caso del costo oportunidad específicamente desde la perspectiva del paciente.

Medir en la embarazada el costo oportunidad a partir del tiempo empleado en la búsqueda y obtención de la atención y relacionar este indicador con el costo del tiempo medido a partir de la remuneración económica, pareciera ser una propuesta congruente. No obstante se puede cuestionar la estimación del costo del tiempo para la población que no percibe remuneración económica. Sin embargo, adoptar el costo promedio del tiempo a partir del ingreso de la población económicamente activa, o de la inversión en los escolares o en la guardería parece ser la opción que mejor refleja la realidad.

Calcular el costo oportunidad del traslado, espera y atención pretende capturar la realidad que existe en torno a la búsqueda y obtención de la atención en una mujer embarazada, no se puede negar que desde la perspectiva de la paciente estos tres momentos son los que rodean a la atención médica. Lo que es materia de discusión es la similitud del costo oportunidad del traslado y la espera en la Consulta de Medicina Familiar, así como la distancia que se amplia en relación a la espera en Laboratorio y Farmacia. Esto lo que refleja es la importancia de acercar los servicios de salud al lugar donde reside la población. Junto a ello es necesario comentar la relación entre el costo oportunidad del traslado y espera, con el costo oportunidad de la atención, este último es menor que los dos primeros. Al respecto se puede decir que el acto médico, la relación médico paciente en la cual se establece el diagnóstico, la evaluación y el plan de manejo, tiene un costo oportunidad menor al costo oportunidad del resto de las acciones que se requieren para lograr la atención, esto puede resultar de inicio contradictorio y absurdo, no obstante parece corresponder a la realidad de la práctica de la medicina pública y privada. 

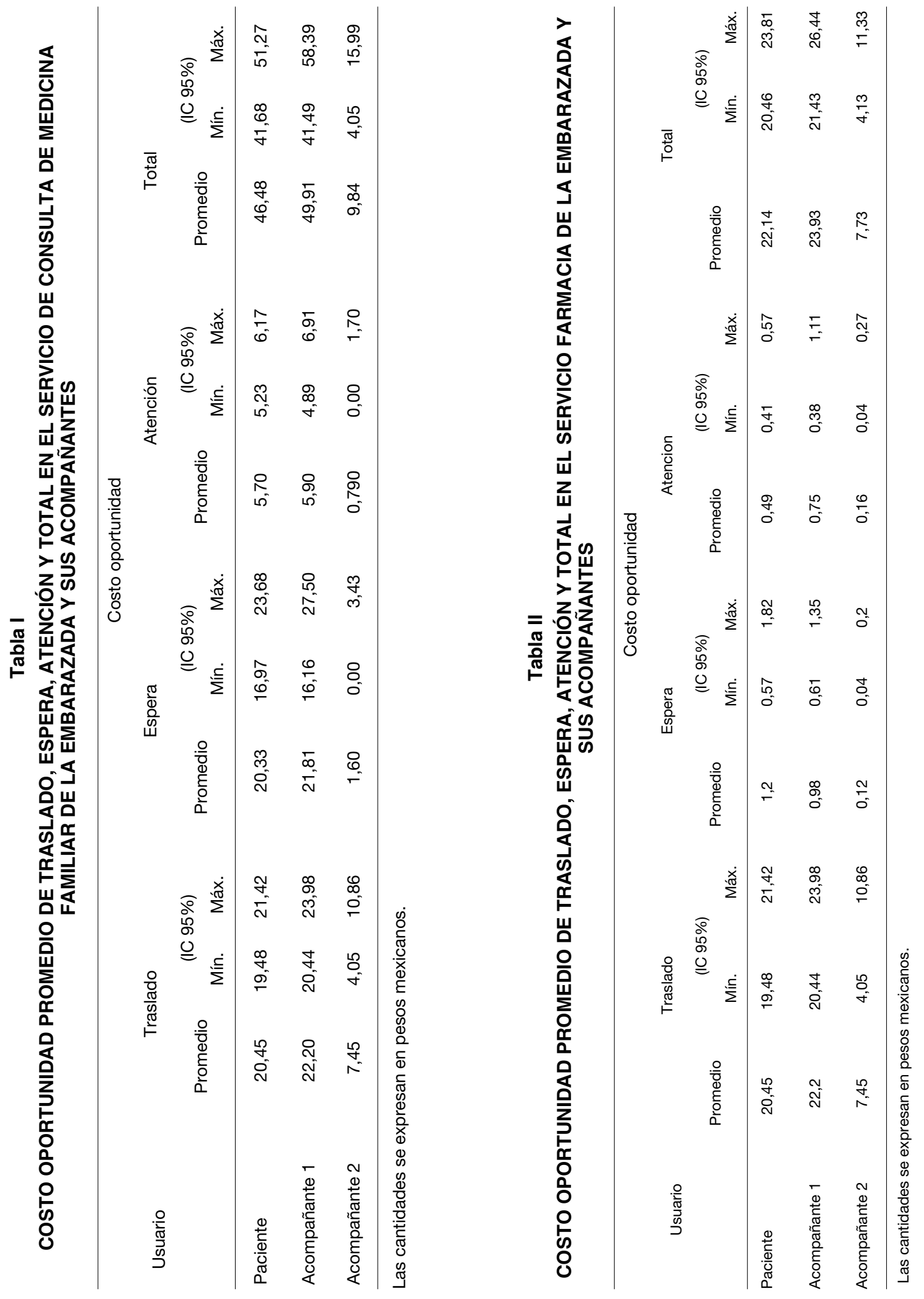


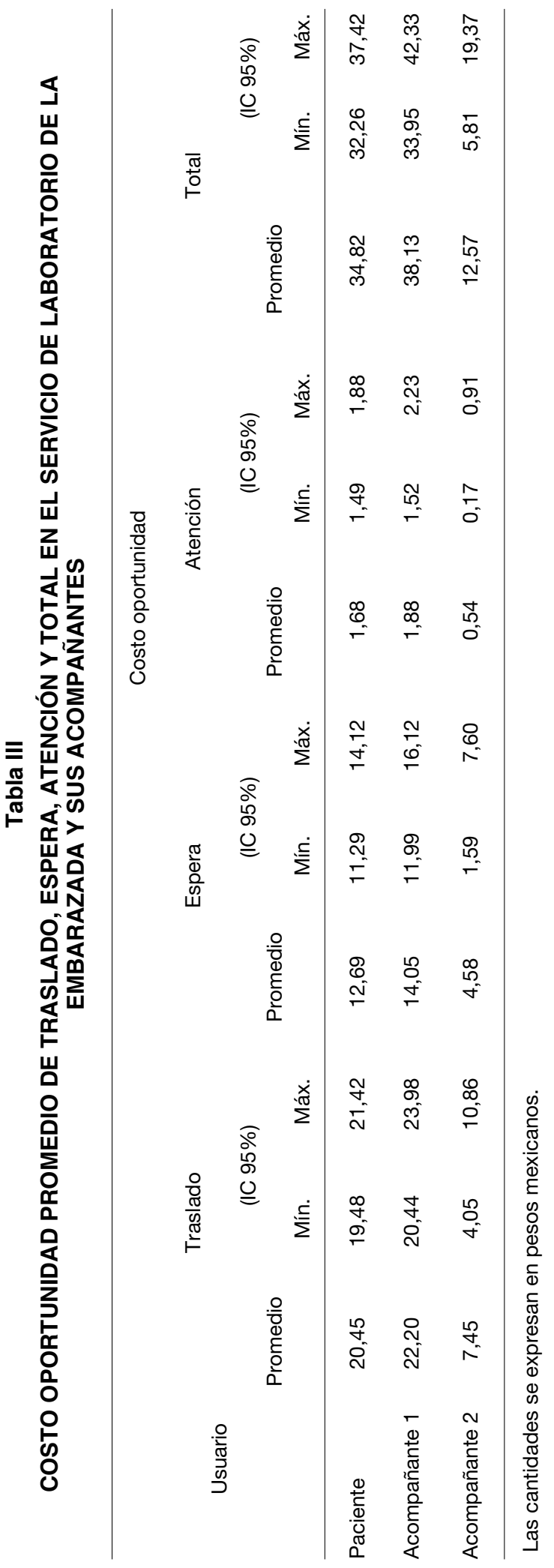




\section{Tabla IV}

\section{COSTO OPORTUNIDAD PROMEDIO POR TIPO DE ESCENARIO DE LA PACIENTE EMBARAZADA Y SUS ACOMPAÑANTES}

\begin{tabular}{|c|c|}
\hline Tipo de escenario & $\begin{array}{l}\text { Costo oportunidad } \\
\text { promedio }\end{array}$ \\
\hline \multicolumn{2}{|c|}{ PACIENTE SOLO } \\
\hline Consulta de Medicina Familiar & 46,48 \\
\hline Farmacia & 22,14 \\
\hline Laboratorio & 34,82 \\
\hline Consulta de Medicina Familiar y Farmacia & 48,17 \\
\hline Consulta de Medicina Familiar y Laboratorio & 60,85 \\
\hline Farmacia y Laboratorio & 36,51 \\
\hline Consulta de Medicina Familiar, Farmacia y Laboratorio & 62,54 \\
\hline \multicolumn{2}{|c|}{ PACIENTE Y ACOMPAÑANTE 1} \\
\hline Consulta de Medicina Familiar & 95,62 \\
\hline Farmacia & 44,29 \\
\hline Laboratorio & 75,52 \\
\hline Consulta de Medicina Familiar y Farmacia & 99,81 \\
\hline Consulta de Medicina Familiar y Laboratorio & 126,69 \\
\hline Farmacia y Laboratorio & 76,37 \\
\hline Consulta de Medicina Familiar, Farmacia y Laboratorio & 130,11 \\
\hline \multicolumn{2}{|c|}{ PACIENTE, ACOMPAÑANTE 1 Y ACOMPAÑANTE 2} \\
\hline Consulta de Medicina Familiar & 106,23 \\
\hline Farmacia & 53,80 \\
\hline Laboratorio & 85,52 \\
\hline Consulta de Medicina Familiar y Farmacia & 109,93 \\
\hline Consulta de Medicina Familiar y Laboratorio & 141,65 \\
\hline Farmacia y Laboratorio & 89,22 \\
\hline Consulta de Medicina Familiar, Farmacia y Laboratorio & 145,35 \\
\hline
\end{tabular}

El costo oportunidad más alto en Consulta de Medicina Familiar que en Laboratorio y este mayor que en Farmacia, se establece en función del costo oportunidad de la espera, lo cual podría controlarse con el uso de visitas programadas en las cuales se limitara el tiempo de espera.

Incorporar al estudio el comportamiento de la población que acude sola o con acompañantes también se establece con la intención de capturar la realidad de la población embarazada. Es una verdad la presencia de los acompañantes, ya sean adultos o menores, en este caso los hijos, la im- plicación estriba en el costo oportunidad que se genera por parte del acompañante, lo cual en teoría incrementa el costo, pero también es necesario destacar la ganancia secundaria por la presencia de los acompañantes.

En este contexto se debe destacar el costo oportunidad más alto del primer acompañante, el cual generalmente corresponde a una persona adulta con actividad remunerada. Ya se ha descrito que la contribución económica de la mujer se estima insuficientemente debido a que se inserta en el sector informal de la economía, no obstante esta es 
Tabla V

COSTO OPORTUNIDAD PROMEDIO AJUSTADO POR TIPO DE ESCENARIO DE LA PACIENTE EMBARAZADA Y SUS ACOMPAÑANTES

\begin{tabular}{lc}
\hline Tipo de escenario & $\begin{array}{c}\text { Costo oportunidad } \\
\text { promedio ajustado }\end{array}$ \\
\hline $\begin{array}{l}\text { Consulta de Medicina } \\
\text { Familiar }\end{array}$ & 77,14 \\
Farmacia & 37,08 \\
Laboratorio & 58,64 \\
Consulta de Medicina & \\
Familiar y Farmacia & 79,89 \\
Consulta de Medicina & \\
Familiar y Laboratorio & 101,46 \\
Farmacia y Laboratorio & 61,39 \\
Consulta de Medicina & \\
Familiar, Farmacia y & \\
Laboratorio & 104,20
\end{tabular}

Las cantidades se expresan en Pesos Mexicano

una realidad que presenta una tendencia al cambio (14). Este mismo panorama es total opuesto cuando se analiza el ingreso del segundo acompañante que en la mayoría de los casos corresponde a escolar.

Conocer el porcentaje de embarazadas que acuden solas o con uno o dos acompañantes permite estimar el costo oportunidad ajustado. La importancia de éste radica en la posibilidad de establecer un valor único promedio para los servicios estudiados y en consecuencia estar en posibilidad de extrapolar este al resto de la población, ello en la búsqueda de identificar el comportamiento poblacional y la planeación de los servicios de salud.

\section{CONCLUSIÓN}

El costo oportunidad en la paciente embarazada, que demanda atención en primer nivel, tiene variaciones importantes, las cuales están determinadas por el número de servicios que utilice y la presencia de acompañantes.

\section{BIBLIOGRAFÍA}

1. Sanabria RG, Gálvez GA, Álvarez MM. Costos para la mujer durante la atención prenatal. Rev Cubana de Salud Pública 2004; 30(7):77-82.

2. Sepúlveda AC. Eficiencia e ineficiencia del gasto en salud. Chile, 1990-1999. Una reflexión metodológica. Cuad Méd Soc 2004;44:251-64. [Citada 2009 May 6]. Hallado en: http://www.colegiomedico.cl/Portals/0/files/biblioteca/publicaciones/cuadernos/44_4.pdf

3. Baly GA, Toledo ME, Rodríguez JF. La economía de la salud, la eficiencia y el costo de oportunidad. Rev Cubana Med Gen Integr 2001;17(4):395-8.

4. Arredondo A, Nájera P, Leyva R. Atención médica ambulatoria en México: el costo para los usuarios. Salud Pública Mex 1999;41(1):18-26.

5. Puig-Junoy J, Pinto PJ. El coste de oportunidad del tiempo remunerado en la producción de salud. Centre de Recerca en Economa i Salut Departament d'Economia i Empresa Universitat Pompeu Fabra 2001:1-30. [citada 2009 Mar 9]. Hallado en: http:// www.econ.upf.es/ puig/publicacions/paper39.pdf

6. Fernández-Valenzuela Y. Métodos de evaluación económica aplicadas a salud. [citada 2009 May 8]. Hallado en: http://www.odontomarketing.com/articulos/art30.htm

7. Informe al Ejecutivo Federal y al Congreso de la Unión sobre la situación financiera y los riesgos del Instituto Mexicano del Seguro Social 2007- 2008. [Citada 2009 May 6]. Hallado en: http://www.imss.gob.mx/instituto/ informes/informe_ejecutivo_2007_2008.htm

8. Instituto Nacional de Estadística y Geografía INEGI. Total de embarazos en México 2005. [Citada 2009 May 7]. Hallada en: http://www.inegi.org.mx/est/contenidos/espanol/proyectos/conteos/conteo2005/sintesis.pps

9. Instituto Mexicano del Seguro Social. Número de consultas a embarazadas. Sistema de Información Médico Operativa (SIMO) IMSS, Querétaro, Qro; Instituto Mexicano del Seguro Social, 2008.

10. Garza Elizondo ME, Rodríguez Casas S, Núñez Rocha GM, Rodríguez López O, Villarreal Ríos E. Perfil de uso de los servicios de salud en primer nivel de atención por mujeres embarazadas. En: Villarreal RE, Martínez GL Galicia RL, Vargas DER. El sistema de salud, perfil de uso y costo en el primer nivel de atención. Primera Edición, Querétaro, México. Universidad Autónoma de Querétaro 2007, 52-8.

11. Reportes interactivos. Sistema de Información de Estadística en la Educación Básica. [citado 2009 Abr 19]. Hallado en: http://sieeb.basica.sep.gob.mx/\#

12. 12.- Guarderías. Capítulo 1. El IMSS en la Seguridad Social. Instituto Mexicano del Seguro Social [edición electrónica]. 2001 [citado 2009 Abr 19]; 10:[aprox. 15 pág.]. Hallado en: http://www.imss. gob.mx/NR/rdonlyres/D36A95CC-1CDD-41D7-A48561AB747EAB22/0/CAP1.pdf

13. Porcentaje de inflación anualizada de países americanos seleccionados. [citado 2009 Ago 3]. Hallado en: http://www.mexicomaxico.org/Voto/Inflacion.htm

14. Cómputo del costo de la desigualdad de género en: United Nations Population Fund. El estado de la población mundial 2000. [citado 2009 Ago 3] Hallado en: http://www.unfpa.org/swp/2000/espanol/ch05.html 